\title{
A Virtual Environment based Serious Game to Support Health Education
}

\author{
Tiago Gomes ${ }^{1}$, Tiago Abade ${ }^{1}$, José Creissac Campos ${ }^{1}$, Michael D. Harrison ${ }^{2}$, José Luís Silva ${ }^{3, *}$ \\ ${ }^{1}$ Departamento de Informática/Universidade do Minho \& HASLab/INESC TEC, Braga, Portugal \\ ${ }^{2}$ Newcastle University \& Queen Mary University of London, UK \\ ${ }^{3}$ Madeira-ITI, Universidade da Madeira, Funchal, Portugal
}

\section{Abstract}

APEX was developed as a framework for ubiquitous computing (ubicomp) prototyping through virtual environments. In this paper the framework is used as a platform for developing a serious game designed to instruct and to inform. The paper describes the Asthma game, a game aimed at raising awareness among children of asthma triggers in the home. It is designed to stimulate a healthier life-style for those with asthma and respiratory problems. The game was developed as the gamification of a checklist for the home environment of asthma patients.

Received on 01 December 2013; accepted on 03 February 2014; published on 04 March 2014

Keywords: Human Factors, Design, Health Education, Serious Games, Asthma, Virtual Environments

Copyright (c) 2014 Tiago Gomes et al., licensed to ICST. This is an open access article distributed under the terms of the Creative Commons Attribution license (http: / / creativecommons . org/licenses/by/3.0/), which permits unlimited use, distribution and reproduction in any medium so long as the original work is properly cited.

doi:10.4108/amsys.1.3.e5

\section{Introduction}

Serious games support playing to learn. Games can be used to promote training, education, health, public policies and strategic communication as well as to provide pleasure [1-3]. This paper is concerned with the possibility of promoting health education through computer-based serious games.

The paper describes a first person game that addresses the problems faced by children with asthma. Asthma is a chronic disease and specific procedures prevent the emergence of crises. The goal of the game is to convey knowledge about these procedures, focusing children at elementary school level. The game was designed as the gamification [4] of a checklist used to evaluate the living conditions of asthma sufferers.

First person games typically involve control of an avatar that is placed inside a $3 \mathrm{D}$ virtual world. The paper illustrates the use of a framework called APEX, that is designed to prototype ubicomp environments [5], to support the rapid development of serious games. Ubicomp environments provide personalized services to users within physical spaces through the integration of environmental information using sensors. APEX

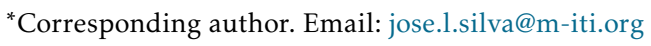

supports the creation of virtual environments (using a 3-D application server) and the definition of behaviors within them, and can be used to develop games within these environments.

The paper extends work originally presented in [6], providing a more detailed description of the rationale behind the game's design. It also describes an evaluation of the first version of the game and proposals for design improvement.

\section{Related work}

A substantial research literature is concerned with exploring how best to design serious games for health education and training. An extensive review of the general area of video games in health care can be found in [7]. This review spans education and training to therapeutic applications of games. The more specific topic of immersive 3-D environments in healthcare education is also reviewed in [8].

3-D application servers, that can be used to develop the kind of immersive 3-D game that is the focus of the paper, have also been explored in other work. Boulos provides an overview of the use of Second Life [9]. Two of their conclusions are: (1) that educators need to think out of the box when using this type of environment, 


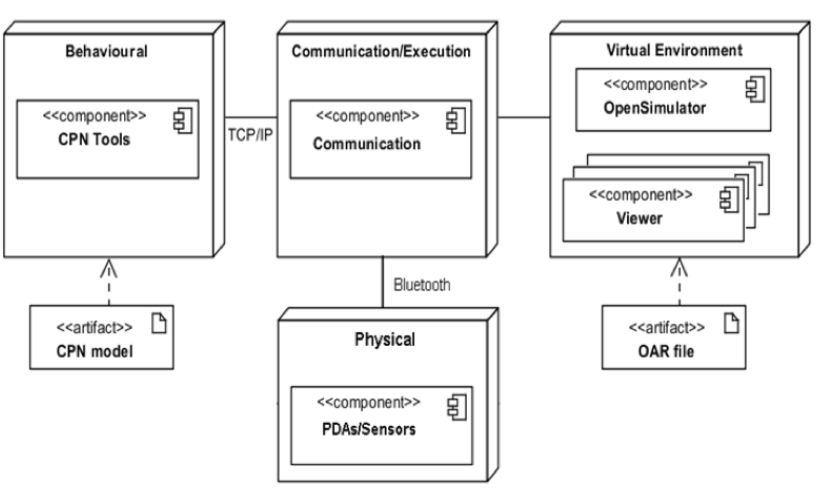

Figure 1. APEX Architecture

avoiding a replication of the class room context inside the virtual environment, and (2) that research is still needed to make 3-D virtual worlds more accessible and to improve the efficiency and effectiveness of their use for learning and teaching. The present paper addresses these two issues. Gamification provides a means of making environments more engaging than a simple replication of the physical world. APEX provides a flexible means to develop such games.

\section{The APEX framework}

APEX (rApid Prototyping for user EXperience) [5] is a framework for the rapid prototyping of ubicomp environments, enabling their simulation and analysis in the early stages of development. The platform consists of a number of components providing support for prototyping at different levels. There are four main components (see logical architecture in Figure 1).

(1) The behavioral component (top left in the figure) is responsible for managing the behavior of the prototype. It is based on CPN Tools ${ }^{1}$ and uses Coloured Petri Nets (CPN) [10] to describe the behavior of the virtual environment in response to user actions and context changes.

(2) The virtual environment component (top right) is responsible for managing the physical appearance and layout of the prototype. It is based on OpenSimulator ${ }^{2}$, a multi-platform and multi-user virtual environment simulator.

(3) The communication/execution component (top middle) and (4) the physical component (bottom middle) are responsible for coordinating the communication between all components and for communication with external devices, respectively. These components form an OpenSimulator module.

\footnotetext{
${ }^{1}$ CPN Tools: http: / / cpntools .org (last visited: 29/11/2013)

2Opensimulator: http://opensimulator.org/ (last visited: 29/11/2013)
}

The platform supports different types of evaluation of the prototypes using these components. The models of the behavioral component can be analyzed. The developed prototype can be used to evaluate user experience experimentally.

This paper describes how APEX was used to create a virtual environment that incorporates a serious game (cf. [11]). The aim of the proposed game is to convey information about asthma and how best to prevent asthma attacks.

\section{Asthma}

Asthma is a chronic inflammatory disease of the respiratory tract characterized by variable and recurring symptoms, reversible airflow obstruction, and bronchospasm. The most common symptoms include wheezing, coughing and shortness of breath [12].

Asthma attacks can arise for a number of reasons. The most common reasons are drug intake while eating or taking medication, and inhaling substances such as pollen, smoke, animal dander or dust. Many of the substances that cause asthma attacks derive from the presence of mites. These substances are often present in the home. Upholstery, curtains and clothes often harbor large communities of mites.

There are several procedures that prevent the causes of asthma attacks, but these procedures are not always known by asthma sufferers. Parents, and especially children, need support to identify what triggers asthma so that they can take appropriate action. Government and non-government organizations have developed checklists that aim to provide such support. The EPA $^{3}$ Asthma Home Environment Checklist [13] is one example. The checklist covers six allergens and irritants (Dust Mites, Pests, Warm-blooded Pets, Mold, Secondhand Smoke and Nitrogen Dioxide), as well as mentioning a generic class of chemical irritants. For each allergen/irritant the checklist identifies triggers as well as typical locations where it can be found. The checklist contains 23 questions divided into two categories distinguishing Home interior and Room interior. Each question is associated with action that can be taken.

Examples of Home interior questions include "Does anyone smoke in the home or car", "Is the patient's asthma worse when around warm-blooded pets?" and "Are there air conditioning window units?".

Examples of Room interior questions include: "What does the patient sleep on?", "Are stuffed toys present?" and "What window coverings are present?"

An illustration of action steps proposed by the checklist relating to warm-blooded pets is the following:

\footnotetext{
${ }^{3}$ The United States Environmental Protection Agency.
} 


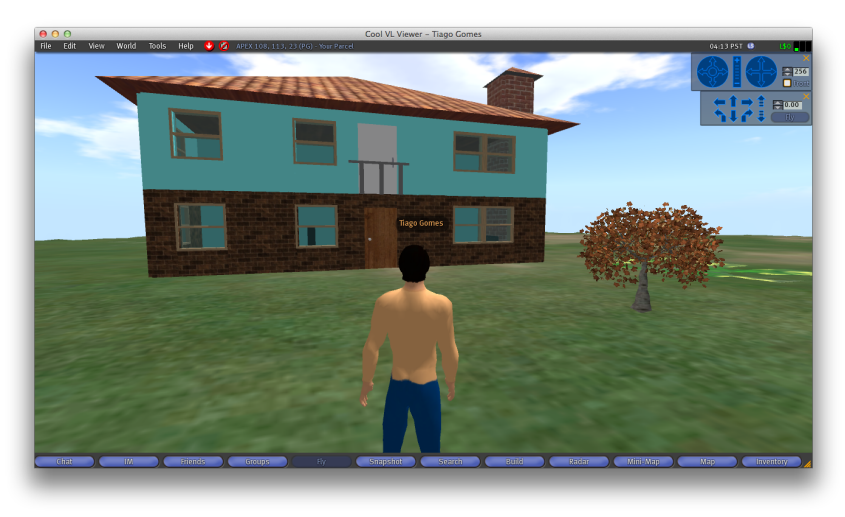

Figure 2. The Asthma game's house (outside view)

- If possible, remove the pet from the home or keep the pet outside.

- If this is not possible, keep the pet out of the patient's sleeping area and off the furniture.

Checklists, however, are not the best way to help children learn how to avoid asthma attacks. The EPA checklist was originally designed for home care visitors, rather than children or parents. The aim of the game is to convey the information contained in the EPA checklist more attractively to parents and children. As a result it is to be hoped that quality of life will be improved and dependency on home care visits reduced.

\section{Design of the Asthma Game}

The APEX platform was used to develop a 3D immersive environment that aims to allow players to connect the information provided in the checklist to their daily lives. A more technical description of how APEX was used to build the game is provided in [14]. Here the focus of the discussion is on how the game was derived from the EPA checklist.

Since the checklist offers information about the interior of homes, the game was set in a house (see Figure 2). The house used (derived from a model of the Aware Home at the Georgia Institute of Technology [15]) is a two storey building containing rooms and areas typically found in a home.

The first version of the game addresses six of the allergens and irritants identified in the EPA checklist: Dust Mites, Warm-Blooded Pets, Mold, Nitrogen Dioxide, chemical irritants and second hand smoke. Pests were not addressed. One or more situations were created that represented the presence of the allergen/irritant in question, providing a potential asthma trigger.

Nine situations covered the first five of these allergens/irritants.

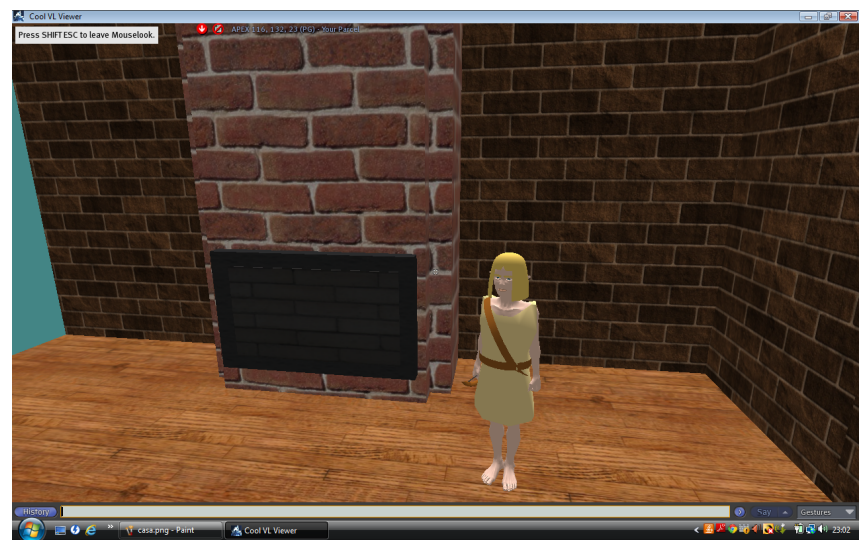

Figure 3. A fire place in the house

1. Domestic pets were placed inside the house, more specifically in a bedroom. This was done to illustrate the second question in the checklist ("Is the patient's asthma worse when around warmblooded pets?").

2. Laundry was littered around some of the floor areas, thereby offering threat of Dust Mites. While this feature did not relate directly to the checklist, it was considered relevant given the target group for the game.

3. A fireplace was created in the house (see Figure 3) that would generate Nitrogen Dioxide. This feature illustrated the checklist item: "Are supplemental heating sources used?".

4. Stuffed toys were provided, particularly in the bedroom. This was another situation relating to Dust Mites and addressed the checklist question: "Are stuffed toys present?".

5. Some of the walls in the house had accumulated mold. Relevant checkist questions here were: "Is there evidence of water damage, moisture, or leaks (such as damp carpet or leaky plumbing)?" and "Do you see or smell mold or mildew (such as in the bathroom on tub, shower, walls, or windows)?".

6. Curtains were used in the house, again relating to Dust Mites and the question:"What window coverings are present?".

7. Blankets were provided on beds, again relating to Dust Mites, and to the question "What types of bedding does the patient use?".

8. Cleansing products were placed in easily accessible places, for example the bathroom (see Figure 4). This related to the question "Is the patient's asthma worse when around chemicals or products 


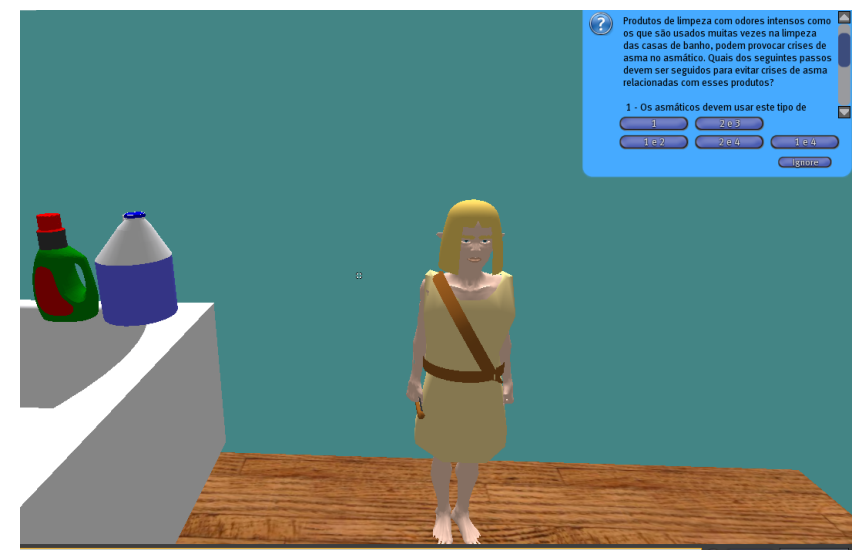

Figure 4. Cleansing products in the bathroom

with strong odors (such as cleaners, paints, adhesives, pesticides, air fresheners, or cosmetics)?".

9. Carpets were provided on the floor. This occurrence further related to dust mites and the question:"What type of floor covering is present?".

No explicit situation was created for second hand smoke. However, as explained below, this allergen's presence was addressed outside the house.

A quiz game was conceived to convey information about actions relating to these situations. A NonPlayer Character (NPC) was placed next to each trigger (see Figures 3 and 4). These characters were designed to provide relevant information about "their" allergen/irritant. They were also designed to ask players relevant questions about how to act to avoid asthma attacks. A further NPC was placed at the entrance of the house to deal with the secondhand smoke allergen, dealing generically with this situation.

Each player controls an avatar in the virtual world (see Figure 2). They can interact with NPCs by approaching and explicitly touching them (allowing interaction with the object in the virtual environment). Players can also see and interact with each other (e.g. by exchanging messages). Each player is allowed to continue to answer a question until the right answer is found. For each correct answer, the player gets a word which, at the end of the game, can be used to form a sentence about asthma. This is intended as an incentive for players to attempt to answer all the questions. After answering the questions, the player is notified that the game has ended, and of how many wrong answers were given. If playing competitively, the first player to correctly answer all questions wins.

As far as is possible the phrasing of the questions is similar to that of the EPA checklist. However, some adaptations were made to accommodate the age range of the target audience for the game. As an illustration the question relating to cleansing products, see item 8 above, was expressed as (translated from the original Portuguese formulation used in the game and illustrated in Figure 4):

- "Cleansing products with intense odors, such as those often used in the cleaning of toilets, can cause asthma attacks. Which of the following steps should be followed to prevent asthma attacks related to these products?"

The NPC puts forward four possible answers to choose from. Correct answers capture action steps listed in the checklist. Wrong answers correspond to actions that go against what is recommended. Because the checklist offers more than one action step for each question, it is possible that more than one correct answer can be provided by a player. The correct answers must therefore be chosen from a set of alternatives.

For example, take the case of chemicals and products with strong odors (specific examples are cleansing products) the checklist proposes the following action steps:

- "Limit patient's exposure as much as possible by minimizing product use, using products only when patient is not present, or trying alternative products."

- "If products are used, carefully follow manufacturer's instructions on the label and make sure the area is well ventilated."

This was translated into the following four possible answers/actions:

1. Asthmatics should use this type of product

2. The use of these products should be avoided or minimized, if possible replacing them with other products

3. If their use is unavoidable, they should be used when the asthmatic person is not in the house

4. This type of product is never harmful for asthmatics

The player must then identify the correct answers from this set of alternatives.

\section{User Study}

A usability study was designed to assess the acceptability of the game as well as its potential as a means of learning about asthma. Participants in the study were aged 9 and 10 years, attending the fourth year of primary school. All participants were already in possession of a personal computer ${ }^{4}$ with enough features and

\footnotetext{
${ }^{4}$ Intel Classmate-based laptop computers.
} 
capabilities to run the game. Their machines were preinstalled with the software needed to play the game (the Cool VL Viewer ${ }^{5}$ ).

Initially, a study had been planned to compare a group of children playing the game with a group attending a regular lesson about Asthma. When discussing the study with the teaching staff, however, two concerns were raised. Only half of the children would play the game. It was felt that this would be unfair to the other half, and could make them feel less motivated. At the same time it was felt that comparing the game against a particular teacher would not necessarily represent traditional teaching practice in general.

It was therefore decided to evaluate the children's perception of what they had learned, an indirect measure of evaluating the game that follows the approach taken in $[16,17]$ for example.

18 children (11 boys and 7 girls) were chosen to play. The study took place in a classroom. None of the children had previous experience of 3D application servers (e.g. Second Life $^{6}$ ). Twelve children stated that they had played computer games before (this information was obtained through a questionnaire - see below). The session began with instructions designed to enable all participants to use the platform without problems. They were allowed time to familiarize with the Cool VL Viewer and the virtual environment. The participants were given a minimum of 30 minutes to complete the Asthma game. During this period they were observed by three evaluators. Their teacher was also present.

The children were asked to answer questions as requested by the NPCs when touching them. The game finished when all questions were correctly answered by the player. Wrong answers were counted and the results presented at the end. At that point, using the words won by each right answer, the player should be able to form the sentence about asthma.

Apart from two computers that had initial problems accessing the APEX server, all users were quickly engaged in the game. Their engagement was of two types. Some children actually played the game, trying to answer the questions. A majority, however, became more interested in exploring the virtual environment and interacting with other players through it. This interaction could be observed both as chatting within the virtual environment, and pushing or running into other avatars. Some children complained of not being able to play the game as a result of this type of interference.

\footnotetext{
${ }^{5}$ Cool VL Viewer: http://sldev.free.fr/ (last visited: 29/12/2013)

${ }^{6}$ Second Life: http://secondlife.com/ (last visited: 29/12/2013)
}

Exploration of the virtual environment progressed through stages. Initially, players realized they could walk into the water surrounding the island where the house was set and could explore the seabed. Then they realized that they could activate a flight mode and fly around in the environment (a particularly popular activity). These two features were the main distractions. They caused a few players to lose their way so that they required help to return to the island and the house. At a later stage, a reduced number of players realized they could manipulate the virtual environment by creating (later also deleting) objects.

In consequence, while reaction to the game was positive, some of available features of the virtual environment contributed to distraction from the purpose of the game.

After playing the game, each player completed a 3-point Likert scale questionnaire ${ }^{7}$ describing their experience. The data collected helped complement and extend the analysis derived from observation. Results show that players enjoyed the game. For example, 16 out of 18 players found the game fun to play, and 12 found it easy to play. None gave negative replies to these questions. In terms of perception of what the children had learnt, despite the observed distractions during game play, the results were positive (with the mode of all questions being the Yes reply). 12 children felt the game had helped them to know more about the disease and to be able to help others. 14 felt they now knew what to do at home to prevent respiratory problems (one child gave a negative answer to this question), and all felt motivated to apply what they had learnt.

In summary, results indicate that the game can help in conveying information about asthma, but some improvements were needed to guarantee that players stay focussed on the game's objectives.

\section{Game redesign}

A second version of the game was developed to overcome some of the problems with the first version. This new version preserves the rules and mechanics of the game. Changes were mainly concerned with the set up of the virtual environment.

A transparent barrier was placed between land and water, and the flight mode was disabled. These limitations made it possible to prevent both entry into water and distraction caused by the flight functionality. Since a significant number of players lost time changing the environment, the functionalities that enabled construction and editing of the environment were disabled. The chat functionality was not disabled as it was felt that some degree of interaction between players is useful.

\footnotetext{
${ }^{7}$ Available at: http://ivy.di.uminho.pt/apex
} 
At this stage we are preparing a second study with the new version of the game. Preliminary results indicate that the new configuration of the virtual environment succeeded in focussing players on the game. A number of preliminary test subjects have been able to complete the game successfully.

\section{Conclusions and future work}

Serious Games combine learning with entertainment. As part of the APEX project an approach was developed to enable the rapid development of prototypes of ubicomp environments. This paper describes how the environment was used for the development of a serious game that addresses the problems faced by children with asthma.

The goal of the game is to impart knowledge about how to act when faced with factors that might cause asthma attacks. The game was designed as a gamification of the EPA Asthma Home Environment Checklist. Action steps in the checklist were transformed into answers to questions that addressed the items in the checklist. The game does not cover all aspects of the checklist (it would become too long). However the advantage of using APEX is that new versions can be created quickly that address further different aspects of the checklist.

To validate the concept, a user study was conducted. This study revealed that the game (its virtual environment) succeeded in capturing the attention of children. Additionally, it showed that children felt they had learnt about the disease while having fun playing the game. However, the study also showed that there is a need to restrict what avatars can do in the environment, to better focus the players on the goals of the game. Several features of Opensimulator were new to children and may explain the distraction. A second version of the game was developed that avoids the problematic features of the virtual environment. Preliminary results based on evaluation of the new version are positive, indicating that this version succeeds in focusing the players' attention.

In conclusion, while virtual environments prove engaging for activities of this kind, there is a need to configure them appropriately to ensure adherance to the objectives of the game. The development of both versions of the game demonstrate that the APEX framework is a feasible and flexible approach to the rapid development of serious games.

\section{Acknowledgements}

This work was carried out in the context of the APEX project, funded by ERDF - European Regional Development Fund through the COMPETE Programme (operational programme for competitiveness) and by
National Funds through the FCT - Fundação para a Ciência e a Tecnologia (Portuguese Foundation for Science and Technology) within project FCOMP-010124-FEDER-015095.

\section{References}

[1] Авт, С.C. (1970) Serious games (The Viking Press).

[2] ZydA, M. (2005) From visual simulation to virtual reality to games. IEEE Computer 38(9): 25-32.

[3] Mouaheb, H., Fahli, A., Moussetad, M. and Eljamali, S. (2012) The serious game: What educational benefits? Procedia - Social and Behavioral Sciences 46: 5502 - 5508.

[4] Marczewski, A. (2013) Gamification: A Simple Introduction, 2nd ed.

[5] Silva, J.L., Ribeiro, O.R., Fernandes, J.M., Campos, J.C. and Harrison, M.D. (2010) The APEX framework: prototyping of ubiquitous environments based on petri nets. In Human-Centred Software Engineering (Springer), Lecture Notes in Computer Science 6409: 6-21.

[6] Gomes, T., Abade, T., Harrison, M., Silva, J. and Campos, J. (2013) Developing serious games with the APEX framework. In Proc. Wksp. Ubiquitous games and gamification for promoting behavior change and wellbeing: 37-40.

[7] Kato, P.M. (2010) Video games in health care: Closing the gap. Review of General Psychology 14(2): 113-121.

[8] Hansen, M.M. (2008) Versatile, immersive, creative and dynamic virtual 3-D healthcare learning environments: A review of the literature. Journal of Medical Internet Research 10(3): e26.

[9] Boulos, M., Hetherington, L. and Wheeler, S. (2007) Second life: an overview of the potential of 3-D virtual worlds in medical and health education. Health Information E Libraries Journal 24(4): 233-245.

[10] Jensen, K. and Kristensen, L.M. (2009) Coloured Petri Nets (Springer).

[11] de Freitas, S. (2008) Serious Virtual Worlds: a scoping study. Tech. Rep. 480, JISC.

[12] Akinbami, L.J. and Schoendorf, K.C. (2002) Trends in childhood asthma: Prevalence, health care utilization, and mortality. Pediatrics 110(2): 315-322.

[13] EPA, U. (2004), Asthma Home Environment Checklist, United States Environmental Protection Agency.

[14] Gomes, T., Abade, T., Campos, J., Harrison, M. and SiLva, J. (2014) Rapid development of first person serious games using the apex platform: The asthma game. In ACM SAC 2014. Accepted.

[15] Kientz, J.A., Patel, S.N., Jones, B., Price, E., Mynatt, E.D. and AвоwD, G.D. (2008) The Georgia Tech aware home. In CHI '08 Extended Abstracts (ACM): 3675-3680.

[16] Mendonça, C., Sousa, N., Soares, P., Varajão, I. and Oliveira, J. (2012) The role of motion interaction in learning mathematical tasks in a computer game. In Proc. 5th Intl. Conf. of Education, Research and Innovation (ICERI 2012) (IATED): 1370-1376.

[17] Fernandes, A., Sousa, N., Soares, P., Noy, D., Varajão, I. and Oliveira, J. (2013) Type of task in a mathematical skills videogame affects children's perception of learning and amusement. In Proc. 7th Intl. Technology, Education and Development Conf. (INTED 2013) (IATED): 515-522. 